\title{
Comparative Persistence of Methotrexate and Tumor Necrosis Factor Inhibitors in Rheumatoid Arthritis, Psoriatic Arthritis, and Ankylosing Spondylitis
}

\author{
Michael D. George (D), Joshua F. Baker (D), and Alexis Ogdie (D)
}

ABSTRACT. Objective. The role of methotrexate (MTX) for the treatment of spondyloarthritis (SpA) remains uncertain. Aims were to compare MTX and tumor necrosis factor inhibitor (TNFi) persistence in spondyloarthritis versus rheumatoid arthritis (RA) and to determine whether concomitant conventional synthetic disease-modifying antirheumatic drug (csDMARD) use is associated with improved TNFi persistence in SpA.

Methods. This retrospective cohort study using Optum's deidentified Clinformatics Data Mart Database 2000-2014 identified patients with RA, psoriatic arthritis (PsA), and ankylosing spondylitis (AS) without prior biologic use who were initiating MTX or a TNFi. Cox proportional hazards models compared time to medication discontinuation over the next 2 years between patients with RA, PsA, or AS, adjusting for potential confounders. In similar analyses stratified by disease, Cox models were used to assess whether concomitant use of csDMARD was associated with TNFi persistence. Results. We identified 31,527 MTX initiators (26,708 RA, 2939 PsA, 1880 AS) and 34,651 TNFi initiators (24,134 RA, 6705 PsA, 3812 AS). MTX was discontinued sooner in patients with PsA [adjusted HR (aHR) 1.10, 95\% CI 1.04-1.16] and AS (aHR 1.23, 1.16-1.31) versus RA, while TNFi were discontinued at similar rates in RA and AS and discontinued later in PsA (aHR 0.93, 0.89-0.97). Concomitant use of MTX (compared to no csDMARD) was associated with lower rates of TNFi discontinuation in RA (aHR 0.85, 0.80-0.89), PsA (aHR 0.81, 0.74-0.89), and AS (aHR $0.79,0.67-0.93)$.

Conclusion. MTX discontinuation occurs sooner in patients with PsA and AS versus RA. Concomitant use of MTX with a TNFi, however, is associated with improved TNFi persistence in all 3 diseases. (First Release April 1 2020; J Rheumatol 2020;47:826-34; doi:10.3899/jrheum.190299)

Key Indexing Terms: SPONDYLOARTHRITIS ANTIRHEUMATIC DRUGS TUMOR NECROSIS FACTOR INHIBITORS METHOTREXATE

Methotrexate (MTX) is the backbone of therapy for the treatment of rheumatoid arthritis (RA), but the role of MTX in the treatment of spondyloarthropathies (SpA) such as

From the University of Pennsylvania, Division of Rheumatology, and Perelman School of Medicine, Department of Biostatistics, Epidemiology, and Informatics; Philadelphia Veterans Affairs Medical Center, Division of Rheumatology, Philadelphia, Pennsylvania, USA.

This work was supported by the McCabe Foundation; the Rheumatology Research Foundation Scientist Development Award to MG; the US National Institute of Arthritis and Musculoskeletal and Skin Diseases at the National Institutes of Health (grant numbers K23 AR073931-01 to MG and R01 AR072363 to AO); and the US Veterans Affairs Clinical Science Research and Development (grant number I01 CX001703 to $J B)$. Dr. Ogdie has received research grant funding to the University of Pennsylvania from Novartis and Pfizer.

M.D. George, MD, MSCE, Instructor, University of Pennsylvania, Division of Rheumatology; J.F. Baker, MD, MSCE, Assistant Professor, Philadelphia VA Medical Center, Division of Rheumatology, and University of Pennsylvania; A. Ogdie, MD, MSCE, Assistant Professor, University of Pennsylvania, Division of Rheumatology, and Perelman School of Medicine, Department of Biostatistics, Epidemiology, and Informatics.

Address correspondence to Dr. M.D. George, 5 White Building,

3400 Spruce St., Philadelphia, Pennsylvania 19104, USA.

E-mail: Michael.george@uphs.upenn.edu

Accepted for publication August 22, 2019. psoriatic arthritis (PsA) and ankylosing spondylitis (AS) remains unclear. MTX is an effective drug in RA; about $30 \%$ of patients with RA are able to achieve remission with initial therapy with MTX alone ${ }^{1,2,3}$. Studies in PsA and AS are much more limited and have not documented clear benefit. Whether the lack of evidence reflects a lack of efficacy of MTX in SpA, heterogeneity of these diseases, or the lack of adequately powered trials is uncertain. The recent SEAM-PsA trial comparing MTX to etanercept (ETN) in PsA found that a higher proportion of patients had improvements in disease activity with MTX monotherapy than previous studies had reported, but there was no placebo group comparison ${ }^{4}$.

Consequently, the role of MTX and other conventional synthetic disease-modifying antirheumatic drugs (csDMARD) in the treatment of peripheral SpA is a matter for debate (biologics being preferred for axial disease). The European League Against Rheumatism (EULAR) recommends MTX as first-line therapy for PsA $\mathrm{A}^{5}$, but recent guidelines from the American College of Rheumatology (ACR), influenced by the limited data for MTX and large studies of biologics, recommend a tumor necrosis factor inhibitor

Personal non-commercial use only. The Journal of Rheumatology Copyright @ 2020 . All rights reserved. 
(TNFi) as first-line therapy, particularly in patients with more severe disease ${ }^{6}$.

One potential benefit of MTX across all 3 conditions, however, is that combination treatment with a TNFi may prolong or enhance the effectiveness of the TNFi. There is evidence that MTX use can help to prevent the formation of anti-drug antibodies in $\mathrm{RA}^{7}$, but studies in PsA and AS have been mixed ${ }^{8,9,10,11,12}$. Trials, including the recent SEAM-PsA trial, have generally not demonstrated improved efficacy of combining csDMARD treatment with a $\mathrm{TNFi}^{4,8,9,13}$. Because of the shorter duration of trials, however, they are limited in their ability to assess the effects of csDMARD on longer-term TNFi persistence (as opposed to initial efficacy). Some observational studies, on the other hand, have suggested an association between MTX use and TNFi persistence in PsA, even though biologics are the focus of these studies ${ }^{14,15,16}$. Whether to initiate or continue MTX or other csDMARD to influence the persistence of TNFi is an important and common clinical question.

Few studies have examined differences in persistence of MTX and TNFi across RA, PsA, and AS in a real-world setting. Further, few studies have assessed how MTX and other csDMARD affect persistence on a TNFi across these diseases. The objectives of our study were to compare rates of MTX and TNFi discontinuation across diseases and to evaluate whether concomitant use of different csDMARD was associated with TNFi persistence. We hypothesized that patients with PsA and AS would demonstrate reduced persistence of MTX but similar persistence of TNFi (reflecting reduced efficacy of MTX in PsA and AS) and that use of csDMARD with a TNFi would be associated with greater TNFi persistence in RA.

\section{MATERIALS AND METHODS}

Study setting. We conducted a retrospective cohort study using Optum's deidentified Clinformatics Data Mart Database 2000-2014. OptumInsight is a deidentified US administrative claims database from a nationally representative private healthcare insurer, with sociodemographic, inpatient, outpatient, and prescription claims available for over 60 million unique members.

We identified adults $\geq 18$ years old with RA, PsA, or AS based on 2 International Classification of Diseases, 9th ed diagnosis codes on separate days at any time prior to the index date and DMARD use ${ }^{17,18,19}$. We used a hierarchical categorization approach for patients meeting criteria for multiple diseases based on the specificity of these codes (PsA > AS > RA) to create mutually exclusive groups, similar to previous studies ${ }^{20}$. Results were similar if patients meeting criteria for multiple diseases were included within each of these disease categories (non-mutually exclusive groups, not shown).

Patients were indexed at the time they received a first-ever MTX prescription or a first-ever TNFi prescription or infusion (study design in Supplementary Figure 1, available with the online version of this article). We excluded patients with prior biologic use. We required at least 6 months of preceding data (baseline) to ensure that patients were new initiators of their therapy, and we required at least 90 days of available data after medication initiation. We excluded patients with a diagnosis code for systemic lupus erythematosus in the past year or missing demographic data.

Exposures. We first treated disease (RA vs PsA vs AS) as the exposure of interest, comparing rates of MTX or TNFi discontinuation. In subsequent analyses, the exposure of interest was the use of concomitant MTX, sulfasalazine (SSZ), hydroxychloroquine (HCQ), or leflunomide (LEF), defined as a prescription fill 0-90 days after the first TNFi prescription fill or infusion. In this analysis, exposure to one of these csDMARD was compared to no csDMARD use.

Outcomes. The outcome of interest was the time to medication discontinuation within 2 years of starting MTX or a TNFi. We selected 2 years as the cutoff based on the distribution of time to therapy discontinuation and the distribution of time in the cohort. Courses of therapy were identified by using days of supply for prescriptions and set intervals for procedure codes [e.g., 56 days for infliximab (IFX) and golimumab (GOL) infusions, 30 days for certolizumab pegol (CZP) procedure codes], truncating for early prescriptions or infusions. The presence of a 90-day gap between the end of 1 prescription/infusion and the start of a new prescription/infusion was defined as a medication discontinuation (the end of a medication course). We also assessed rates of early discontinuation within 90 days of initiation and delayed discontinuation after 90 days of use. For example, a fill of a single 90-day prescription would be counted as early discontinuation.

Covariates. We evaluated a number of covariates that we hypothesized would be associated with medication persistence, including age, sex, calendar year, and other treatments in the 90 days prior to medication initiation (opioids, nonsteroidal antiinflammatory drugs, glucocorticoids, csDMARD). Another covariate was comorbidities based on diagnosis codes in the past year including diabetes, cancer, chronic kidney disease, asthma or chronic obstructive pulmonary disease, congestive heart failure, coronary artery disease, cerebrovascular disease, liver disease, obesity, depression, anxiety, bipolar disorder, chronic pain, peptic ulcer disease, inflammatory bowel disease, as well as the Charlson score with RA codes removed $^{21}$. We also evaluated the number of hospitalizations, emergency department visits, and outpatient visits as a count of events, and included presence of inpatient or outpatient psychiatry visits in the past year. Analyses of TNFi persistence also included type of TNFi as a covariate.

Statistical analysis. Median time to MTX discontinuation or TNFi discontinuation in patients with RA, PsA, or AS was evaluated using Kaplan-Meier methods. Cox proportional hazards models were used to compare time to either MTX or TNFi discontinuation over the next 2 years between patients with RA, PsA, or AS, adjusting for potential confounders. Patients were censored at the soonest of (1) end of health plan enrollment, (2) end of followup (December 31, 2014), or (3) two years after the start of the medication course. Survivor functions were plotted at the mean of all covariates in the models. Delayed discontinuation was assessed in identical Cox proportional hazards models among patients with at least 90 days of MTX or TNFi treatment. Early discontinuation within 90 days was assessed using similar logistic regression models for this binary outcome.

To assess the effect of concomitant csDMARD on TNFi persistence, we evaluated patients with at least 90 days of TNFi treatment to allow a 90-day window after TNFi initiation to measure prescriptions for concomitant csDMARD. In this population, we evaluated time to TNFi discontinuation within 2 years using Cox models. Here analyses were stratified by disease (RA, PsA, and AS) with separate models for each of the csDMARD, comparing rates of TNFi discontinuation in patients receiving each csDMARD to rates of TNFi discontinuation in patients receiving no concomitant csDMARD. The same covariates were used in all models. To avoid overfitting these models, we selected covariates from a Cox model of delayed TNFi discontinuation including patients with RA, PsA, and AS, using stepwise backward deletion of covariates with $\mathrm{p}>0.1$, forcing age, sex, calendar year, type of TNFi, and glucocorticoid use into the models. We assessed interactions between disease and concomitant csDMARD use in models including patients with all 3 diseases as a 3-level variable. To assess heterogeneity in effect by type of TNFi, we also assessed interactions between type of TNFi and concomitant csDMARD. We performed additional sensitivity analyses in cases where interactions were observed.

All analyses were conducted using STATA version 15.0 (StataCorp.).

Personal non-commercial use only. The Journal of Rheumatology Copyright @ 2020 . All rights reserved. 
Because this study used a deidentified dataset, the study was deemed exempt from ethical review by the University of Pennsylvania Institutional Review Board.

\section{RESULTS}

Basic demographics. Among 44,897 patients with RA, PsA, and AS initiating MTX and 60,763 initiating a first TNFi, 31,527 MTX initiators (26,708 RA, 2939 PsA, 1880 AS) and 34,651 TNFi initiators (24,134 RA, 6705 PsA, 3812 AS) met all inclusion and exclusion criteria (Figure 1). The most common TNFi were ETN $(16,405)$ and adalimumab (9592), followed by IFX (6734), GOL (1031) and CZP (880). Patients with RA were more frequently treated with csDMARD and glucocorticoids. Opioid use, depression, and chronic pain were frequent in all patient groups, with the highest rates in patients with AS (Table 1). Median available followup time was 2.0 years (IQR 1.0-3.9) in the MTX cohort and 2.0 years (IQR 1.0-3.9) in the TNFi cohort.

MTX and TNFi discontinuation rates in RA, PsA, and AS. Patients remained on MTX a median of 1.07 years, with 7581 $(24.0 \%)$ of patients discontinuing treatment within 90 days and 1- and 2-year drug survival rates of $51.4 \%$ and $36.6 \%$, respectively. MTX was discontinued sooner in patients with PsA [adjusted HR (aHR) 1.10, 1.04-1.16] and AS (aHR
1.23, 1.16-1.31) versus RA (Table 2, Figure 2). There were greater rates of both early and delayed MTX discontinuation in patients with PsA and especially AS (Table 3).

Overall, patients continued taking TNFi a median of 1.31 years, with 6065 patients $(17.5 \%)$ discontinuing treatment within 90 days. One and 2-year drug survival rates were $56.1 \%$ and $40.5 \%$, respectively. TNFi discontinuation occurred slightly later in PsA (aHR 0.93, 0.89-0.97) when compared with RA. Patients with AS had similar rates of discontinuation compared to RA (aHR 1.00, 0.94-1.05; Table 2, Figure 2). Similar results were observed for early and delayed discontinuation (Table 3 ).

Concomitant csDMARD use and rates of TNFi discontinuation. Among patients who continued a TNFi for at least 90 days, 12,874/19,903 (64.7\%) with RA, 2307/5604 (41.2\%) with PsA, and 888/3079 with AS (28.8\%) received a concomitant csDMARD in the 90 days after TNFi initiation, most commonly MTX.

Concomitant use of MTX (compared to no csDMARD) was associated with lower rates of TNFi discontinuation in RA (aHR 0.85, 0.80-0.89), PsA (aHR 0.81, 0.74-0.89), and AS (aHR 0.79, 0.67-0.93; Figure 3). SSZ was associated with lower rates of TNFi discontinuation in RA and PsA but
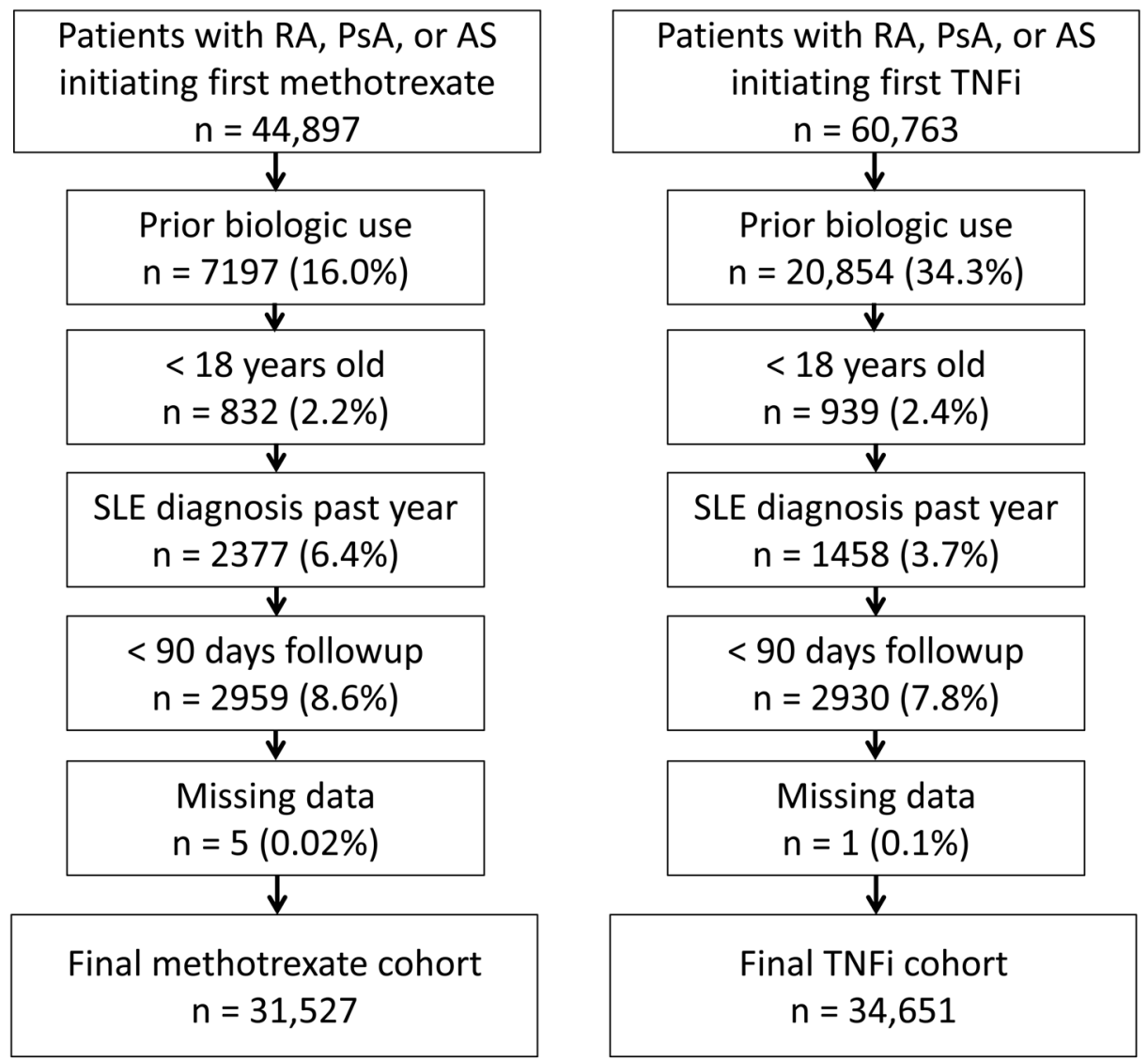

Figure 1. Cohort identification flow diagram. RA: rheumatoid arthritis; PsA: psoriatic arthritis; AS: ankylosing spondylitis; TNFi: tumor necrosis factor inhibitor; SLE: systemic lupus erythematosus. 
Table 1. Patient characteristics.

\begin{tabular}{|c|c|c|c|c|c|c|}
\hline \multirow[t]{2}{*}{ Characteristics } & \multicolumn{3}{|c|}{ MTX Initiators } & \multicolumn{3}{|c|}{ TNF Initiators } \\
\hline & $\mathrm{RA}, \mathrm{n}=26,708$ & PsA, $n=2939$ & AS, $n=1880$ & $\mathrm{RA}, \mathrm{n}=24,134$ & PsA, $n=6705$ & $\mathrm{AS}, \mathrm{n}=3812$ \\
\hline Age, yrs, mean (SD) & $56.8(14.2)$ & $52.3(13.1)$ & $53.9(14.30)$ & $52.7(13.1)$ & $48.9(12.2)$ & $45.9(12.9)$ \\
\hline Previous csDMARD, n (\%) & $10,459(39.2)$ & $744(25.3)$ & $687(36.5)$ & $20,160(83.5)$ & $4498(67.1)$ & $1839(48.2)$ \\
\hline \multicolumn{7}{|l|}{ Treatments past 90 days, $\mathrm{n}(\%)$} \\
\hline MTX & N/A & N/A & N/A & $12,799(53.0)$ & $2768(41.3)$ & $766(20.1)$ \\
\hline Leflunomide & $744(2.8)$ & $44(1.5)$ & $21(1.1)$ & $2686(11.1)$ & $274(4.1)$ & $106(2.8)$ \\
\hline Glucocorticoids & $11,423(42.8)$ & $641(21.8)$ & $663(35.3)$ & $11,020(45.7)$ & $1535(22.9)$ & $1065(27.9)$ \\
\hline NSAID & $8679(32.5)$ & $988(33.6)$ & $655(34.8)$ & $7064(29.3)$ & $2022(30.2)$ & $1365(35.8)$ \\
\hline Opiates & $7917(29.6)$ & $657(22.4)$ & $817(43.5)$ & $7390(30.6)$ & $1611(24.0)$ & $1435(37.6)$ \\
\hline \multicolumn{7}{|l|}{ Comorbidities, $\mathrm{n}(\%)$} \\
\hline Diabetes & $3867(14.5)$ & $412(14.0)$ & $280(14.9)$ & $2896(12.0)$ & $852(12.7)$ & $324(8.5)$ \\
\hline CAD & $2951(11.0)$ & $249(8.5)$ & $206(11.0)$ & $1926(8.0)$ & $431(6.4)$ & $224(5.9)$ \\
\hline Cerebrovascular disease & $969(3.6)$ & $55(1.9)$ & $89(4.7)$ & $601(2.5)$ & $95(1.4)$ & $73(1.9)$ \\
\hline Liver disease & $854(3.2)$ & $110(3.7)$ & $126(6.7)$ & $978(4.1)$ & $380(5.7)$ & $186(4.9)$ \\
\hline Obesity & $1813(6.8)$ & $222(7.6)$ & $162(8.6)$ & $1502(6.2)$ & $523(7.8)$ & $255(6.7)$ \\
\hline Depression & $3460(13.0)$ & $357(12.1)$ & $416(22.1)$ & $3145(13.0)$ & $845(12.6)$ & $616(16.2)$ \\
\hline Anxiety & $2216(8.3)$ & $242(8.2)$ & $257(13.7)$ & $1824(7.6)$ & $584(8.7)$ & $399(10.5)$ \\
\hline Bipolar disorder & $181(0.7)$ & $19(0.6)$ & $34(1.8)$ & $131(0.5)$ & $62(0.9)$ & $38(1.0)$ \\
\hline Chronic pain & $5795(21.7)$ & $447(15.2)$ & $698(37.1)$ & $4576(19.0)$ & $1082(16.1)$ & $1025(26.9)$ \\
\hline Peptic ulcer disease & $465(1.7)$ & $38(1.3)$ & $43(2.3)$ & $437(1.8)$ & $76(1.1)$ & $77(2.0)$ \\
\hline IBD & $290(1.1)$ & $21(0.7)$ & $71(3.8)$ & $630(2.6)$ & $78(1.2)$ & $427(11.2)$ \\
\hline Charlson score & $1[0-3]$ & $0[0-2]$ & $1[0-3]$ & $0[0-2]$ & $0[0-2]$ & $0[0-2]$ \\
\hline \multicolumn{7}{|l|}{ Hospitalizations, n (\%) } \\
\hline
\end{tabular}

MTX: methotrexate; TNF: tumor necrosis factor; RA: rheumatoid arthritis; PsA: psoriatic arthritis; AS: ankylosing spondylitis; csDMARD: conventional synthetic disease-modifying antirheumatic drug; HCQ: hydroxychloroquine; N/A: not available; NSAID: nonsteroidal antiinflammatory drugs; COPD: chronic obstructive pulmonary disease; CHF: congestive heart failure; CAD: coronary artery disease; IBD: inflammatory bowel disease.

Table 2. Rates of MTX and TNFi persistence in patients with RA, PsA, and AS.

\begin{tabular}{|c|c|c|c|c|c|}
\hline \multicolumn{6}{|l|}{ MTX } \\
\hline RA & 26,708 & 23,773 & 1.13 & Ref & Ref \\
\hline AS & 1880 & 1378 & 0.70 & $1.35(1.28-1.44)^{*}$ & $1.23(1.16-1.31)^{*}$ \\
\hline \multicolumn{6}{|l|}{ TNFi } \\
\hline RA & 24,134 & 23,062 & 1.28 & Ref & Ref \\
\hline
\end{tabular}

* $\mathrm{p}<0.05$. Adjusted HR (aHR) from multivariable Cox models adjusted for age, sex, year, prescriptions for opioids, NSAID, MTX (TNF analyses only), sulfasalazine, hydroxychloroquine, leflunomide use in the past 3 months; diabetes, cancer, chronic kidney disease, asthma/chronic obstructive pulmonary disease, congestive heart failure, coronary artery disease, cerebrovascular disease, liver disease, obesity, depression, anxiety, bipolar disorder, chronic pain, peptic ulcer disease, Charlson score, hospitalizations in the past year, emergency room visits in the past year, no. outpatient visits, and psychiatric visits. TNFi models also adjusted for which TNFi patients received. MTX: methotrexate; TNFi: tumor necrosis factor inhibitor; RA: rheumatoid arthritis; PsA: psoriatic arthritis; AS: ankylosing spondylitis; NSAID: nonsteroidal antiinflammatory drugs.

Personal non-commercial use only. The Journal of Rheumatology Copyright $($ C 2020. All rights reserved. 

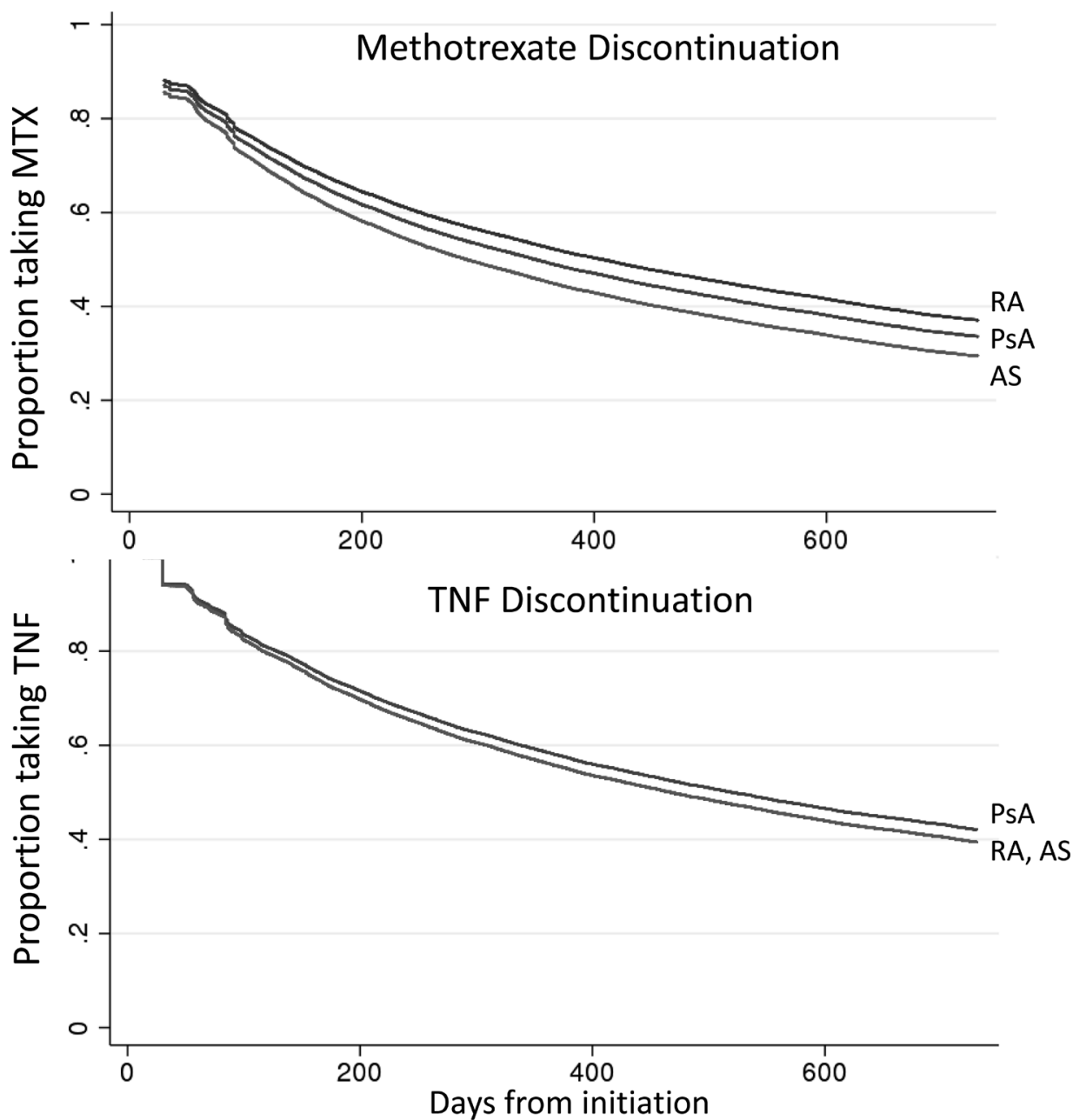

Figure 2. Time to MTX or TNFi discontinuation in rheumatoid arthritis (RA), psoriatic arthritis (PsA), and ankylosing spondylitis (AS). Survivor function from Cox regression models at the means of all covariates: age, sex, year, diabetes, cancer, chronic kidney disease, asthma/chronic obstructive pulmonary disease, congestive heart failure, coronary artery disease, cerebrovascular disease, liver disease, obesity, depression, anxiety, bipolar disorder, chronic pain, peptic ulcer disease, Charlson score, hospitalizations in the past year, emergency department visits in the past year, number of outpatient visits, psychiatry visits, as well as prescriptions for opioids, NSAID, MTX (TNF analyses only), sulfasalazine, hydroxychloroquine, and leflunomide use in the past 3 months. TNFi models also adjusted for which TNFi patients received. NSAID: nonsteroidal antiinflammatory drugs; TNFi: tumor necrosis factor inhibitor; MTX: methotrexate.

not AS. HCQ was associated with significantly lower rates of TNFi discontinuation only in RA. LEF was not associated with lower rates of TNFi discontinuation in RA, PsA, or AS (Figure 3).

There was a significant interaction between type of TNFi and csDMARD use only for MTX $(\mathrm{p}<0.01)$, with evidence of a stronger association between MTX use and IFX discontinuation compared to other TNFi: aHR 0.73 (0.65-0.81) in RA, aHR $0.77(0.60-0.97)$ in PsA, and aHR $0.70(0.49-1.00)$ in AS among IFX-treated patients. We repeated analyses evaluating concomitant csDMARD use in patients treated with non-IFX TNFi and found similar results (Supplementary Figure 2, available with the online version of this article).

\section{DISCUSSION}

This large study using deidentified administrative claims data is one of the first to demonstrate reduced persistence of MTX in patients with PsA and AS compared to patients with RA. In contrast, rates of TNFi discontinuation were similar across the groups. Additionally, we evaluated associations between csDMARD use and TNFi discontinuation, and found that the concurrent use of MTX was similarly associated with lower discontinuation rates for TNFi in RA, PsA, and AS.

MTX discontinuation was more common in PsA and AS compared to RA, with greater rates of both early discontinuation (which might be expected to reflect differences in side effects or toxicity, such as hepatotoxicity) and delayed 
Table 3. Rates of early and delayed MTX and TNFi discontinuation in patients with RA, PsA, and AS.

\begin{tabular}{|c|c|c|c|c|c|c|c|c|}
\hline & \multirow[b]{2}{*}{$\mathrm{n}$} & \multicolumn{3}{|c|}{ Early DC } & \multicolumn{4}{|c|}{ Delayed DC } \\
\hline & & Early DC, n (\%) & OR & $\mathrm{aOR}$ & Person-yrs & $\begin{array}{r}\text { Median } \\
\text { Persistence, }\end{array}$ & HR & aHR \\
\hline \multicolumn{9}{|l|}{ MTX } \\
\hline RA & 26,708 & $6270(23.5)$ & Ref & Ref & 17,993 & 1.88 & Ref & Ref \\
\hline PsA & 2939 & $726(24.7)$ & $1.07(0.98-1.17)$ & $1.06(0.97-1.17)$ & 1842 & 1.61 & $1.16(1.09-1.23)^{*}$ & $1.13(1.06-1.20)^{*}$ \\
\hline AS & 1880 & $585(31.1)$ & $1.47(1.33-1.63)^{*}$ & $1.32(1.20-1.48)^{*}$ & 990 & 1.35 & $1.29(1.20-1.40)^{*}$ & $1.18(1.09-1.27)^{*}$ \\
\hline \multicolumn{9}{|l|}{ TNFi } \\
\hline $\mathrm{RA}$ & 24,134 & $4231(17.5)$ & Ref & Ref & 17,584 & 1.85 & Ref & Ref \\
\hline PsA & 6705 & $1101(16.4)$ & $0.92(0.86-0.99)^{*}$ & $0.91(0.84-0.98)^{*}$ & 5044 & 2.08 & $0.91(0.88-0.95)^{*}$ & $0.95(0.91-1.00)$ \\
\hline AS & 3812 & $733(19.2)$ & $1.12(1.02-1.22)^{*}$ & $1.04(0.94-1.14)$ & 2605 & 1.79 & $1.02(0.97-1.08)$ & $0.99(0.93-1.06)$ \\
\hline
\end{tabular}

$* \mathrm{p}<0.05$. Adjusted OR (aOR) and adjusted HR (aHR) from multivariable logistic or Cox regression models adjusted for age, sex, year, prescriptions for opioids, NSAID, methotrexate (TNF analyses only), sulfasalazine, hydroxychloroquine, leflunomide use in the past 3 months; diabetes, cancer, chronic kidney disease, asthma/COPD, congestive heart failure, coronary artery disease, cerebrovascular disease, liver disease, obesity, depression, anxiety, bipolar disorder, chronic pain, peptic ulcer disease, Charlson score, hospitalizations in the past year, emergency room visits in the past year, no. outpatient visits, and psychiatric visits. TNFi models also adjusted for which TNFi patients received. MTX: methotrexate; TNFi: tumor necrosis factor inhibitor; DC: discontinuation; RA: rheumatoid arthritis; PsA: psoriatic arthritis; AS: ankylosing spondylitis.

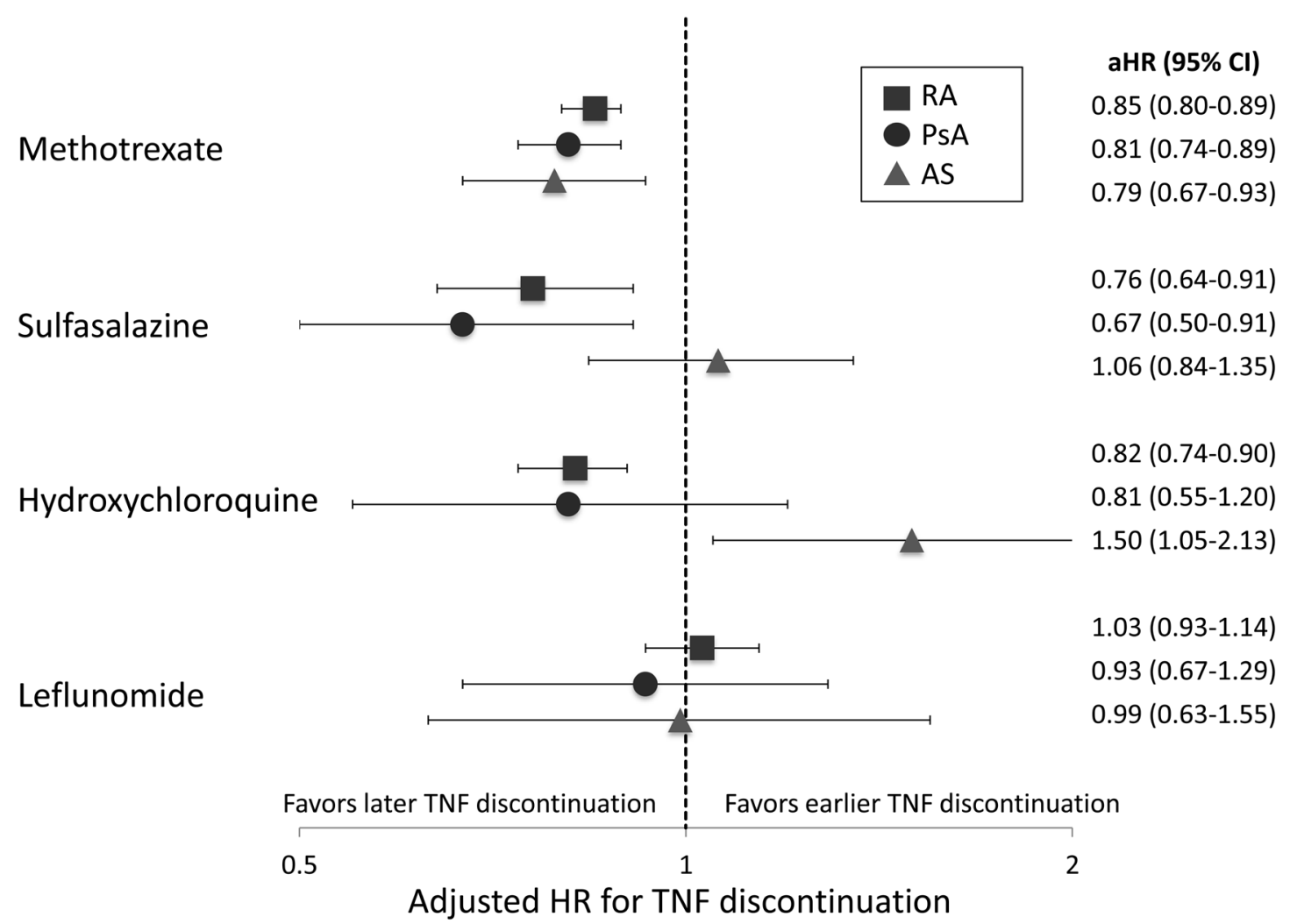

Figure 3. Association between conventional DMARD use and TNFi discontinuation in rheumatoid arthritis (RA), psoriatic arthritis (PsA), and ankylosing spondylitis (AS). Adjusted HR (aHR) from separate Cox models created for each medication, stratified by disease (RA, PsA, or AS). Models also included age, sex, year, type of TNFi, glucocorticoid use, NSAID, depression, bipolar disorder, chronic pain, Charlson score, inflammatory bowel disease, asthma/chronic obstructive pulmonary disease, liver disease, and hospitalizations in the past year. Interactions to assess differences in csDMARD effects across diseases from models with all 3 diseases combined: disease*methotrexate $p=0.62$, disease* sulfasalazine $p=0.03$, disease*hydroxychloroquine $\mathrm{p}<0.01$, disease*leflunomide $\mathrm{p}=0.77$. TNFi: tumor necrosis factor inhibitor; NSAID: nonsteroidal antiinflammatory drugs; csDMARD: conventional synthetic disease-modifying antirheumatic drugs.

discontinuation (presumably more affected by treatment efficacy). The similar rates of TNFi discontinuation in RA, PsA, and AS suggest that the MTX results are not due to greater rates of discontinuation of all medications in SpA. Although the magnitude of difference at a population level was modest, it should be noted that many fewer patients

Personal non-commercial use only. The Journal of Rheumatology Copyright @ 2020 . All rights reserved. 
with SpA initiated MTX compared to the number initiating a TNFi. Patients with SpA who were receiving MTX were a select group that the treating physician might have considered the most likely to respond to MTX. Given that neither EULAR nor ACR guidelines recommend MTX for patients with purely axial disease $e^{5,6,22}$, these patients likely have more peripheral predominant disease. Thus, in our study, the persistence of MTX in PsA and AS may in fact be inflated because of this channeling.

The suggestion that MTX may be less effective in the treatment of SpA versus RA is supported by a randomized trial of 221 patients with PsA that did not show a significant difference in ACR20, 28-joint count Disease Activity Score, or joint counts with MTX compared to placebo, although the dose given was generally low (mean dose $<15$ mg weekly) ${ }^{23}$. The more recent SEAM-PsA trial comparing MTX to ETN or combination therapy found that half of treatment-naive patients with PsA initiating MTX achieved an ACR20 response, but there was no placebo comparison and these patients rarely achieved a "deeper" response defined by fulfillment of the minimal disease activity criteria ${ }^{4}$. A previous, smaller observational study from the Norwegian NOR-DMARD registry 2000-2006 comparing MTX use in RA versus PsA found that patients with PsA had smaller improvements in some disease activity measures and in patient-reported outcomes, although 2-year persistence of MTX was similar in RA and PsA ${ }^{24}$. Differences in practice patterns or the smaller number of alternative treatment options available during the time of this study might explain why MTX persistence differed from our results.

Overall, rates of both MTX and TNFi discontinuation were relatively high in our study compared to previously published data, with median TNFi persistence of 1.31 years in TNFi-treated patients and a 1-year retention rate of only $56 \%$. Previous registry studies have shown 1-year retention rates of about $70-80 \%{ }^{14,16,25}$. One of these studies also showed substantially longer TNFi persistence in SpA versus RA, unlike in our study ${ }^{14}$. These differences might be explained by different study populations (registry studies vs a general population study), outcome measurements (patient-report vs prescription data), and differences over time and by country or insurance plan. As more treatment options for SpA have become available, rates of discontinuation would be expected to rise with patients and physicians less willing to accept suboptimal outcomes. Indeed, as in other studies ${ }^{26}$, we found that discontinuation occurred sooner in later calendar years for all diseases (data not shown).

Concomitant use of MTX in patients initiating a TNFi was associated with lower rates of TNFi discontinuation in patients with RA, PsA, and AS, suggesting that MTX may help prolong the efficacy of TNFi in both RA and SpA. While the effect size was largest in patients receiving IFX, the association remained in patients treated with other TNFi. Secondary analyses of clinical trials have not found evidence of greater TNFi efficacy in patients with PsA receiving concomitant $\mathrm{MTX}^{8,13,27}$, and the recent SEAM-PsA trial showed no benefit of ETN with MTX over ETN alone ${ }^{4}$. Previous observational studies, however, have suggested an association between MTX use and longer TNFi persistence ${ }^{14,15,16}$. Discrepancies in these results could be related to inadequate power or shorter duration of clinical trials, differences between clinical trial protocols and real-world treatment, or to confounding in observational studies, with MTX-treated patients perhaps having different health behaviors (healthy adherer effect) or better medication tolerability. Interestingly, in our study we found that the magnitude of the effect of MTX on TNFi discontinuation in PsA and AS was very similar to the magnitude of effect in RA. Given that the efficacy of MTX use with a TNFi has been well established in $\mathrm{RA}^{7}$, similar results in PsA and AS may suggest a similar biologic effect in these diseases. In some studies, MTX has been associated with lower rates of antidrug antibodies in patients with $\mathrm{SpA}$, as has been found in patients with $\mathrm{RA}^{9,10,11,12}$.

Associations between other csDMARD use and TNFi discontinuation rates were more variable across diseases. SSZ use was associated with lower rates of TNFi discontinuation in RA and PsA with a magnitude similar to the effect of concomitant MTX use, suggesting that SSZ might also be effective in promoting TNFi persistence in these diseases. The utility of SSZ in TNFi-treated patients has not been well studied. One previous small study of 117 patients with AS found that SSZ use was associated with lower rates of switching to a second anti-TNFi in patients with pure axial involvement ${ }^{28}$. We were not able to replicate these findings in AS, although we were not able to differentiate patients with pure axial involvement from those with peripheral involvement.

Real-world observational studies have inherent limitations. We were unable to determine reasons for medication discontinuation. While discontinuations, especially after the first 3 months, likely are due to inadequate efficacy, discontinuation because of side effects, patient choice, or tapering as a result of disease control are also possible. Patient and provider expectations of efficacy, influenced by trials and promotion of biologics, may also influence persistence. Disease severity and phenotypes also cannot be collected from these data; patients with PsA and AS who are treated with MTX monotherapy might be expected to have more peripheral predominant and RA-like disease. We would expect this bias to favor overestimation of the benefit of MTX monotherapy, however, because those would be the patients most likely to respond to treatment. We did not have laboratory data or drug antibody levels, and so cannot know whether associations between csDMARD use and TNFi discontinuation rates were mediated by prevention of anti-drug antibodies, synergistic effects of the TNFi on

Personal non-commercial use only. The Journal of Rheumatology Copyright $(\subset) 2020$. All rights reserved. 
disease control, or confounding factors. Medication courses were based on prescription fill data, and it is possible that some patients may fill prescriptions even after stopping their medication. Finally, we did not statistically compare time receiving TNFi versus time receiving MTX because of potential unmeasured confounding by indication.

Strengths of our study include its size, allowing evaluation of multiple different csDMARD and adjustment for potential confounding factors. Additionally, this data source provides real-world data about current discontinuation rates of MTX and TNFi, without limiting evaluation to a population of patients willing to enroll in a registry.

In this large claims data analysis, patients with PsA and AS were more likely to discontinue MTX compared to patients with RA. Rates of TNFi discontinuation, however, were similar in RA, PsA, and AS. In patients treated with a TNFi, use of MTX was associated with lower rates of TNFi discontinuation in RA, PsA, and AS.

\section{ONLINE SUPPLEMENT}

Supplementary material accompanies the online version of this article.

\section{REFERENCES}

1. Singh JA, Saag KG, Bridges SL, Akl EA, Bannuru RR, Sullivan MC, et al. 2015 American College of Rheumatology Guideline for the treatment of rheumatoid arthritis. Arthritis Rheumatol 2016;68:1-26

2. Goekoop-Ruiterman YP, de Vries-Bouwstra JK, Allaart CF, van Zeben D, Kerstens PJ, Hazes JM, et al. Clinical and radiographic outcomes of four different treatment strategies in patients with early rheumatoid arthritis (the BeSt study): a randomized, controlled trial. Arthritis Rheum 2005;52:3381-90.

3. Moreland LW, O'Dell JR, Paulus HE, Curtis JR, Bathon JM, St. Clair EW, et al. A randomized comparative effectiveness study of oral triple therapy versus etanercept plus methotrexate in early, aggressive rheumatoid arthritis: the treatment of Early Aggressive Rheumatoid Arthritis Trial. Arthritis Rheum 2012;64:2824-35.

4. Mease PJ, Gladman DD, Collier DH, Ritchlin CT, Helliwell PS, Liu L, et al. Etanercept and methotrexate as monotherapy or in combination for psoriatic arthritis: primary results from a randomized, controlled phase III trial. Arthritis Rheumatol 2019; 71:1112-24.

5. Gossec L, Smolen JS, Ramiro S, de Wit M, Cutolo M, Dougados $\mathrm{M}$, et al. European League Against Rheumatism (EULAR) recommendations for the management of psoriatic arthritis with pharmacological therapies: 2015 update. Ann Rheum Dis 2016;75:499-510.

6. Singh JA, Guyatt G, Ogdie A, Gladman DD, Deal C, Deodhar A, et al. Special article: 2018 American College of Rheumatology/ National Psoriasis Foundation guideline for the treatment of psoriatic arthritis. Arthritis Rheumatol 2019;71:2-29.

7. Maneiro JR, Salgado E, Gomez-Reino JJ. Immunogenicity of monoclonal antibodies against tumor necrosis factor used in chronic immune-mediated Inflammatory conditions: systematic review and meta-analysis. JAMA Intern Med 2013;173:1416-28.

8. Mease PJ, Gladman DD, Ritchlin CT, Ruderman EM, Steinfeld SD, Choy EH, et al; Adalimumab Effectiveness in Psoriatic Arthritis Trial Study Group. Adalimumab for the treatment of patients with moderately to severely active psoriatic arthritis: results of a double-blind, randomized, placebo-controlled trial. Arthritis Rheum 2005:52:3279-89.
9. Kavanaugh A, Krueger GG, Beutler A, Guzzo C, Zhou B, Dooley LT, et al. Infliximab maintains a high degree of clinical response in patients with active psoriatic arthritis through 1 year of treatment: results from the IMPACT 2 trial. Ann Rheum Dis 2007;66:498-505.

10. Ducourau E, Mulleman D, Paintaud G, Miow Lin DC, Lauféron $\mathrm{F}$, Ternant $\mathrm{D}$, et al. Antibodies toward infliximab are associated with low infliximab concentration at treatment initiation and poor infliximab maintenance in rheumatic diseases. Arthritis Res Ther 2011;13:R105.

11. Plasencia C, Pascual-Salcedo D, Nuño L, Bonilla G, Villalba A, Peiteado D, et al. Influence of immunogenicity on the efficacy of longterm treatment of spondyloarthritis with infliximab. Ann Rheum Dis 2012;71:1955-60.

12. Vogelzang EH, Kneepkens EL, Nurmohamed MT, van Kuijk AWR Rispens T, Wolbink G, et al. Anti-adalimumab antibodies and adalimumab concentrations in psoriatic arthritis; an association with disease activity at 28 and 52 weeks of follow-up. [Internet. Accessed January 13, 2020.] Available from: ard.bmj.com/content/ early/2014/08/12/annrheumdis-2014-205554.abstract

13. Mease PJ, Kivitz AJ, Burch FX, Siegel EL, Cohen SB, Ory P, et al. Etanercept treatment of psoriatic arthritis: safety, efficacy, and effect on disease progression. Arthritis Rheum 2004;50:2264-72.

14. Heiberg MS, Koldingsnes W, Mikkelsen K, Rødevand E, Kaufmann $\mathrm{C}$, Mowinckel $\mathrm{P}$, et al. The comparative one-year performance of anti-tumor necrosis factor alpha drugs in patients with rheumatoid arthritis, psoriatic arthritis, and ankylosing spondylitis: results from a longitudinal, observational, multicenter study. Arthritis Rheum 2008;59:234-40.

15. Kristensen LE, Gülfe A, Saxne T, Geborek P. Efficacy and tolerability of anti-tumour necrosis factor therapy in psoriatic arthritis patients: results from the South Swedish Arthritis Treatment Group register. Ann Rheum Dis 2008;67:364-9.

16. Glintborg B, Østergaard M, Dreyer L, Krogh NS, Tarp U, Hansen MS, et al. Treatment response, drug survival, and predictors thereof in 764 patients with psoriatic arthritis treated with anti-tumor necrosis factor $\alpha$ therapy: results from the nationwide Danish DANBIO registry. Arthritis Rheum 2011;63:382-90.

17. Ogdie A, Alehashemi S, Love TJ, Jiang Y, Haynes K, Hennessy $\mathrm{S}$, et al. Validity of psoriatic arthritis and capture of disease modifying antirheumatic drugs in the health improvement network. Pharmacoepidemiol Drug Saf 2014;23:918-22.

18. Dubreuil M, Peloquin C, Zhang Y, Choi HK, Inman RD, Neogi T. Validity of ankylosing spondylitis diagnoses in The Health Improvement Network. Pharmacoepidemiol Drug Saf 2016;25:399-404.

19. Ford J, MacFarlane LA, Tong A, Kim SC. Identification of psoriatic arthritis using an administrative claims-based algorithm [abstract]. Arthritis Rheumatol 2018;70 Suppl 10:2135

20. Ogdie A, Yu Y, Haynes K, Love TJ, Maliha S, Jiang Y, et al. Risk of major cardiovascular events in patients with psoriatic arthritis, psoriasis and rheumatoid arthritis: a population-based cohort study. Ann Rheum Dis 2015;74:326-32.

21. Gagne JJ, Glynn RJ, Avorn J, Levin R, Schneeweiss S. A combined comorbidity score predicted mortality in elderly patients better than existing scores. J Clin Epidemiol 2011;64:749-59.

22. van der Heijde D, Ramiro S, Landewé R, Baraliakos X, Van den Bosch F, Sepriano A, et al. 2016 update of the ASAS-EULAR management recommendations for axial spondyloarthritis. Ann Rheum Dis 2017;76:978-91.

23. Kingsley GH, Kowalczyk A, Taylor H, Ibrahim F, Packham JC, McHugh NJ, et al. A randomized placebo-controlled trial of methotrexate in psoriatic arthritis. Rheumatology 2012;51:1368-77.

24. Lie E, van der Heijde D, Uhlig T, Heiberg MS, Koldingsnes W, Rødevand E, et al. Effectiveness and retention rates of methotrexate in psoriatic arthritis in comparison with methotrexate-treated patients with rheumatoid arthritis. Ann Rheum Dis 2010;69:671-6.

Personal non-commercial use only. The Journal of Rheumatology Copyright $\subset$ 2020. All rights reserved. 
25. Saad AA, Ashcroft DM, Watson KD, Hyrich KL, Noyce PR, Symmons DP. Persistence with anti-tumour necrosis factor therapies in patients with psoriatic arthritis: observational study from the British Society of Rheumatology Biologics Register. Arthritis Res Ther 2009;11:R52.

26. Mease PJ, Lesperance T, Liu M, Collier DH, Mason M, Deveikis $\mathrm{S}$, et al. Changes in treatment patterns in patients with psoriatic arthritis initiating biologic and nonbiologic therapy in a clinical registry. J Rheumatol 2017;44:184-92.

27. Kavanaugh A, McInnes I, Mease P, Krueger GG, Gladman D, Gomez-Reino J, et al. Golimumab, a new human tumor necrosis factor alpha antibody, administered every four weeks as a subcutaneous injection in psoriatic arthritis: twenty-four-week efficacy and safety results of a randomized, placebo-controlled study. Arthritis Rheum 2009;60:976-86.

28. Shimabuco AY, Gonçalves CR, Moraes JCB, Waisberg MG, Sampaio-Barros PD, Goldenstein-Schainberg C, et al. Sulfasalazine comedication: a predictor of reduced long-term anti-TNF switch in ankylosing spondylitis [abstract]. Arthritis Rheum 2015;67 Suppl 10:2837. 